\title{
A chimaeric plant virus vaccine protects mice against a bacterial infection
}

\author{
Frank R. Brennan, ${ }^{1}$ Linda B. Gilleland, ${ }^{2}$ John Staczek, ${ }^{2}$ Mary M. Bendig, ${ }^{1}$ \\ William D. O. Hamilton ${ }^{1}$ and Harry E. Gilleland, $\mathrm{Jr}^{2}$
}

\author{
Author for correspondence: William D. O. Hamilton. Tel: +44 1223837611 . Fax: +44 1223837604 \\ e-mail: hamiltonw@axisgenetics.co.uk
}

1 Axis Genetics plc, Babraham, Cambridge CB2 4AZ, UK

2 Department of Microbiology and Immunology, Louisiana State University Medical Center, School of Medicine in Shreveport, Shreveport, LA 71130-3932, USA

\begin{abstract}
The plant virus cowpea mosaic virus (CPMV) is an efficient carrier of foreign peptides for the generation of strong humoral immune responses. Peptides derived from both viruses and bacteria are strongly immunogenic when displayed on the surface of CPMV and elicit high titres of peptide-specific antibody. However, the protective effects of antibodies generated using bacterial epitopes in this system have yet to be demonstrated. In this study the ability of chimaeric virus particles (CVPs) to afford protection against bacterial infection was assessed. Immunization of outbred mice with CPMV expressing a peptide derived from outer-membrane protein $F$ of Pseudomonas aeruginosa (CPMV-PAE5) generated high titres of $P$. aeruginosa-specific IgG that opsonized the bacteria for phagocytosis by human neutrophils and afforded protection upon challenge with two different immunotypes of $P$. aeruginosa in a model of chronic pulmonary infection. When examined $8 \mathrm{~d}$ after challenge, CVP-immunized mice had fewer severe lung lesions and fewer bacteria in their lungs compared to mice immunized with wild-type virus. Different levels of protection were seen with CPMV-PAE5 when Freund's or alum adjuvants were used. These studies highlight the ability of CVPs to generate protective immunity against infectious disease agents.
\end{abstract}

Keywords: cowpea mosaic virus, chimaeric virus particle, Pseudomonas aeruginosa, outer-membrane protein $F$, vaccine

\section{INTRODUCTION}

Although live attenuated bacterial (Everest et al., 1995) and viral (Resnick et al., 1995; Rose \& Evans, 1991; Sutter \& Moss, 1992) vaccines as well as naked DNA vaccines (Montgomery et al., 1994) induce stronger and longer-lasting immune responses than conventional killed/subunit vaccines, even in the absence of adjuvant, there are safety concerns over the use of these vaccines (Vlacha et al., 1996; Klinman et al., 1997; WHO, 1990). In particular, persistence or reversion to virulence of the live vaccine strains and integration of the naked DNA vaccine into the host chromosome are major concerns. To circumvent these potential problems, more effective

Abbreviations: CF, cystic fibrosis; CPMV, cowpea mosaic virus; CVP, chimaeric virus particle; FCA, Freund's complete adjuvant; FD, FisherDevlin; FIA, Freund's incomplete adjuvant; KLH, keyhole limpet haemocyanin; NMS, normal mouse serum; OM, outer membrane; PMN, polymorphonuclear leucocyte; wt, wild-type. inactive vaccines are being developed through modification of proteins or peptides by polymerization (Reed et al., 1997), lipidation (Deprez et al., 1996), oxidation/ reduction (Apostolopoulos et al., 1995) and encapsulation (Lovgren et al., 1990). The immunogenicity of proteins can also be enhanced by coupling them to carrier molecules or bacterial proteins (Zhang et al., 1996; Jahn-Schmid et al., 1997), or by producing them in the form of particulate virus-like particles (Adams $e t$ al., 1987; Ball et al., 1998). Furthermore, the use of more effective adjuvants for both mucosal and systemic delivery has resulted in increased immunogenicity of non-replicating vaccines (Gupka \& Siber, 1995; Elson \& Dertzbaugh, 1994).

In an effort to produce effective non-replicating vaccines, we have been examining the vaccine potential of a plant virus, cowpea mosaic virus (CPMV), genetically engineered (Usha et al., 1993; Porta et al., 1994) to express and display foreign peptides on its surface. In this presentation system, termed EPICOAT, peptides of up 
to $\sim 40$ amino acids are incorporated into specific locations in either of the two virus coat proteins. This results in the presentation of 60 copies of the foreign peptide on the surface of each virus particle, circumventing the need to couple the peptides to carrier molecules. The resultant chimaeric virus particles (CVPs) are easily propagated in plants and large amounts of the CVPs (up to $\sim 1 \mathrm{~g}$ per $\mathrm{kg}$ fresh weight of leaves) can be readily extracted. They have the potential for cost-effective manufacture and are not known to infect mammalian cells, thereby circumventing the safety concerns associated with live attenuated bacteria or viruses. A peptide derived from human immunodeficiency disease virus (HIV) gp41 was shown to be highly immunogenic when displayed on CPMV, inducing HIV-neutralizing antibodies in mice (McLain et al., 1995, 1996; Buratti et al., 1998). Also, another F.PICOAT vaccine, presenting a peptide from the VP2 protein of canine parvovirus, was shown to protect mink from a lethal challenge with the canine-parvovirusrelated mink enteritis virus (Dalsgaard et al., 1997). This was the first demonstration of an experimental vaccine produced exclusively in plants conferring protection in the target animal against a viral disease.

More recently, we have shown that peptides from bacterial proteins are also highly immunogenic when displayed on CPMV. A CPMV-based CVP expressing a peptide derived from the fibronectin-binding protein of Staphylococous anreus was shown to elicit high titres of peptide-specific antibody that completely inhibited fibronectin binding (Brennan et al., 1999a). Furthermore, a peptide derived from the outer membrane (OM) protein $\mathrm{F}$ of Pseudomonas aeruginosa elicited high titres of peptide-specific antibody which recognized all seven immunotypes of $P$. aeruginosa and enhanced their phagocytosis by human neutrophils (Brennan et al., 1999b). Despite the immunogenicity of CPMV-expressed bacterial proteins, their ability to confer protective immunity has not been demonstrated.

$P$. aeruginosa is an important opportunistic bacterial pathogen that causes severe infections in immunocompromised humans, including burn patients, the immunosuppressed, and in children with cystic fibrosis (CF). In CF patients, $P$. aeruginosa remains the leading cause of morbidity and mortality (Buret, 1994) due to chronic colonization of the $\mathrm{CF}$ lung. No means are currently available to block the colonization of the $\mathrm{CF}$ lung by $P$. aeruginosa. The development of a vaccine that could successfully accomplish this remains a highly sought-after goal. Among the most promising vaccine candidates for use in this clinical situation are those based on OM protein $F$ of $P$. aeruginosa. In this study, we sought to determine if vaccination using a CVP displaying a peptide derived from the $\mathrm{OM}$ protein $\mathrm{F}$ could provide protection from a bacterial infection. Outbred mice were immunized with the CVP in either Freund's or alum adjuvants and the levels of protection assessed in a clinically relevant model of $P$. aeruginosa infection.

\section{METHODS}

Bacterial strains and culture conditions. The strains of $P$. aeruginosa used in this study were ATCC 27313 (Difco 0-11; FD immunotype 2) and ATCC 27315 (Difco 0-1 ; FD immunotype 4). Both strains were grown at $30^{\circ} \mathrm{C}$ with shaking in $\mathrm{BBL}$ nutrient broth (Becton-Dickinson Microbiology Systems), or on nutrient agar (Difco) plates.

Construction, propagation and purification of CPMV-PAE5 virions. The construction, propagation and purification of the chimaeric CPMV particles (CPMV-PAE5) have been described previously (Brennan et al., 1999b). CPMV-PAE5 expresses a 34-amino acid peptide (NEYGVEGGRVNAVGSGDNATAEGRAINRRVEAEV) inserted between amino acids 98 and 99 of the $\mathrm{L}$ coat protein subunit (in the $\beta \mathrm{E}-\alpha \mathrm{B}$ loop) of CPMV. This sequence comprises $O M$ protein $F$ peptide 18 linked to OM protein $F$ peptide 10 (peptides 18 and 10 in bold) by a short linker sequence and additional $O M$ protein $F$ amino acids either side of peptide 10 . The latter were included to make the peptide more amenable to presentation on the surface of CPMV. Although peptide 18, unlike peptide 10, has not been shown to provide protection from $P$. aeruginosa challenge (Hughes \& Gilleland, 1995), it was found that higher rates of plant infection and virus yield were achieved if peptide 18 was coexpressed with peptide 10 than if peptide 10 was expressed alone. Each virion expresses 60 copies of the OM protein $F 18 / 10$ peptide, and $1 \mu \mathrm{g}$ CVP contains approximately $40 \mathrm{ng}$ tandem peptide. CPMV-PAE5 was shown previously to elicit antibodies only to peptide 10 (Brennan et al., 1999b).

Immunization of mice. Mice (5-week-old female specific pathogen-free outbred ICR mice from Sprague-Dawley) (45-50 per group) were subcutaneously immunized with $100 \mu \mathrm{g}$ of either CPMV-PAE 5 or wild-type CPMV (wtCPMV) in either Freund's complete adjuvant (FCA) $(1: 1$, Sigma) or alum $\left(2.5 \mathrm{mg} \mathrm{m}^{-1}\right.$, aluminium hydroxide concentrate, Pharmaceutical Associates). Booster injections of $10 \mu \mathrm{g}$ virus were administered in the same adjuvant lexcept that Freund's incomplete adjuvant (FIA) replaced FCA] on days 14, 28 and 42. Five mice from each group were culled on each of days 0 , 27,41 and 53 and the sera from mice of each group pooled and stored at $-20^{\circ} \mathrm{C}$ for future analysis. Fourteen days after the last immunization (day 56), all remaining immunized mice (21-28 per group) were challenged with agar beads containing approximately $5 \times 10^{3}$ live $P$. aeruginosa of the Fisher-Devlin (FD) immunotype 2 (FD2) strain (described below). In a second study using the wtCPMV in alum, CPMV-PAE.5 in alum and CPMV-PAE.5 in FCA/FIA groups, and an identical immunization regimen, mice were challenged with the FD4 strain. In a follow-up experiment, a wtCPMV in FCA/FIA and a CPMV-PAE5 in FCA/FIA group were challenged with the FD4 strain, and the results combined with those of the initial experiment.

ELISA for detection of $\boldsymbol{P}$. aeruginosa-specific serum antibody. Pooled sera from each of the groups were examined for titres of IgG antibodies against $P$. aeruginosa FD2 and FD4 by ELISA as described previously (Hughes et al., 1992; Staczek et al., 1998). Briefly, plates (Dynatech; Immunlon 1) were coated with a suspension of whole $P$. aeruginosa (FD2 or FD4) cells prepared according to the method of Abdillahi \& Poolman (1987). Dilutions of sera were incubated on the bacteriacoated plates and bound antibody was detected with alkalinephosphatase-conjugated goat anti-mouse $\operatorname{IgG}$ (Southern Biotechnology Associates) with $p$-nitrophenyl phosphate (Sigma) as the substrate. The absorbance was measured with an 
automated ELISA reader at $405 \mathrm{~nm}$. Results are expressed as endpoint titre, calculated as the inverse of the dilution that gave a higher $A_{405}$ value than twice the standard deviation (SD) above the mean value obtained with a 1:50 dilution of pooled normal mouse sera (NMS).

Assay for opsonic activity of antisera. The ability of pooled antisera from CPMV-PAE5-immunized groups to mediate the uptake of $P$. aeruginosa cells by human polymorphonuclear leucocytes (PMNs) was compared with the ability of wtCPMV-immunized mouse sera to do likewise. As a positive control, sera pooled from 30 mice immunized with OM protein F (Gilleland et al., 1988) were compared to pooled NMS. The assay was performed as described previously (Gilleland et al., 1992). The FD immunotype 2 and 4 strains were used in the assays. Briefly, bacterial cells were mixed with undiluted heat-inactivated sera and incubated with gentle shaking at $37^{\circ} \mathrm{C}$ for $30 \mathrm{~min}$. Human whole blood was added to the mixture and incubated for a further $30 \mathrm{~min}$ at $37^{\circ} \mathrm{C}$. After incubation of the blood with the bacteria and antisera, the mixture was fixed onto slides and stained with Giemsa stain. Each slide was examined microscopically and the number of bacterial cells contained within the first 50 isolated, intact PMNs encountered was determined for each reaction mixture. The assay was performed twice for each of the pooled antisera so that a total of $100 \mathrm{PMNs}$ were counted for each of the antisera. The mean number $( \pm S D)$ of bacterial cells per PMN was then calculated. The statistical significance of the differences noted between groups was evaluated using the unpaired Student's $t$-test, with $P \leqslant 0.05$ considered significant.

Challenge of mice with $\boldsymbol{P}$. aeruginosa. Mice were challenged by using a model of chronic pulmonary infection with $P$. aeruginosa (Gilleland et al., 1988; Staczek et al., 1998). Two weeks after the final immunization, the mice were challenged with agar beads containing $P$. aeruginosa cells of either the FD2 or FD4 immunotype strain. The mice were first anaesthetized with an intraperitoneal injection of sodium pentabarbital and then inoculated via a tracheal incision with $50 \mu \mathrm{l}$ of an agar bead slurry encasing approximately $5 \times 10^{3}$ c.f.u. of $P$. aeruginosa. A beaded-tip 22-gauge needle was gently guided to favour inoculation of the left lung. The incision was closed with sterile wound clips. Eight days after the challenge, the mice were killed by cervical dislocation. Protection conferred on CVP-immunized mice was assessed by two methods. First, the lungs were examined macroscopically for the presence of lesions (Staczek et al., 1998). Lesions were scored as 0 to $4+$ based on the scale detailed in Table 2. An investigator well experienced in macroscopic lung lesion scoring scored the pulmonary lesions. Secondly, the number of $P$. aeruginosa c.f.u. present in the lungs was quantified as described previously (Staczek et al., 1998). Statistical analyses of the differences between wtCPMVimmunized and CPMV-PAE5-immunized groups in scores of lung lesions and number of the bacteria present in lungs were performed with the IBM EpiStat Basic Statistics Program, and $P$ values were calculated by Fisher's exact test. A $P$ value $\leqslant 0.05$ was considered significant.

\section{RESULTS}

\section{CPMV-PAE5 elicits $P$. aeruginosa-specific opsonizing antibody}

Mice immunized with CPMV-PAE5 in either FCA/FIA or alum produced both $P$. aeruginosa FD2- and FD4- (a)
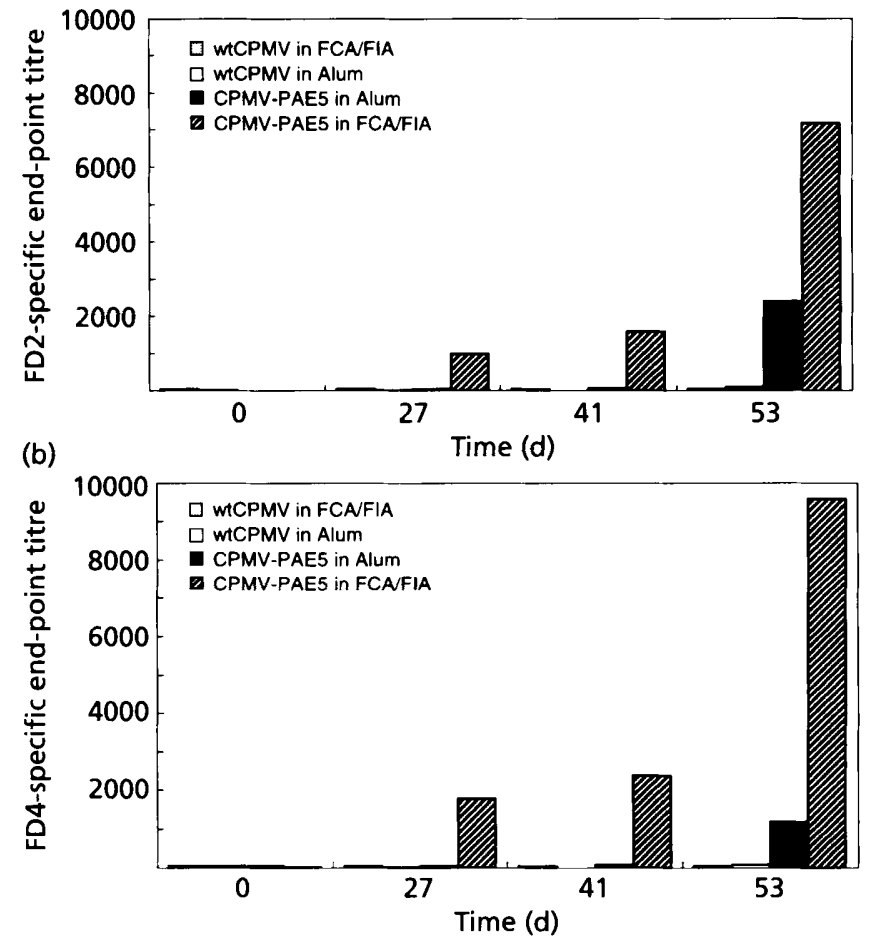

Fig. 1. Antibody responses in immunized mice. Outbred ICR mice were immunized subcutaneously with $100 \mu \mathrm{g}$ of CPMVPAE5 or wtCPMV in either FCA or alum and boosted with $10 \mu \mathrm{g}$ on days 14,28 and 42 with same adjuvant as used for the initial immunization (except that FIA replaced FCA). Sera were collected on days $0,27,41$ and 53 (pre-challenge), pooled and tested for whole $P$. aeruginosa FD2-specific (a) or FD4-specific (b) serum IgG by ELISA. End-point titres obtained with the pooled sera from each group are shown.

specific IgG (Fig. 1a and b, respectively), which was absent in mice immunized with wtCPMV in either alum or FCA/FIA. Immunization with CPMV-PAE5 in Freund's adjuvants elicited higher titres of both FD2 and FD4-specific antibodies than did immunization with CPMV-PAE5 in alum at all time-points examined. CPMV-PAE5 in Freund's adjuvants elicited specific antibody as early as day 27 (after two immunizations) whereas antibody was not detected in mice vaccinated with CPMV-PAE5 in alum until after four immunizations, on day 53 (Fig. 1).

The CPMV-PAE5-immunized mice produced antisera by day 53 that were highly opsonic for $P$. aeruginosa (Table 1). Sera from mice immunized with CPMV-PAE5 in either FCA/FIA or alum were significantly more opsonic for FD2 than sera from wtCPMV-immunized groups. The degree of opsonization (FCA/FIA $>$ alum) paralleled the titres of FD2-specific IgG present in the sera (Fig. 1a). The level of opsonization of FD2 cells observed with sera from mice immunized with purified protein $\mathrm{F}$ in alum was significantly higher (ratio of 1.76) than with sera from CPMV-PAE5-immunized mice when alum was used (ratio of 1.35) but not when 
Table 1. Phagocytic uptake by PMNs of $P$. aeruginosa exposed to the various antisera

\begin{tabular}{|llccc|}
\hline Strain & \multicolumn{1}{c}{ Test serum } & $\begin{array}{c}\text { Mean no. of bacteria } \\
\text { per PMN } \pm \text { SD }\end{array}$ & Ratio $^{*}$ & $\boldsymbol{P}_{\text {value }}$ \\
\hline FD2 & wtCPMV in alum & $9 \cdot 68 \pm 7.23$ & - & - \\
& CPMV-PAE5 in alum & $13.09 \pm 8.49$ & 1.35 & 0.003 \\
& wtCPMV in FCA/FIA & $9 \cdot 83 \pm 9 \cdot 22$ & - & - \\
& CPMV-PAE5 in FCA/FIA & $18.96 \pm 12.36$ & 1.93 & $<0.000001$ \\
& NMS & $11.10 \pm 8.39$ & - & - \\
& Protein F in alum & $19.58 \pm 9.62$ & 1.76 & $<0.000001$ \\
& wtCPMV in alum & $12.97 \pm 7 \cdot 86$ & - & - \\
& CPMV-PAE5 in alum & $17.28 \pm 10.48$ & 1.33 & 0.001 \\
& wtCPMV in FCA/FIA & $13.03 \pm 10.43$ & - & - \\
& CPMV-PAE5 in FCA/FIA & $20.87 \pm 14.60$ & 1.6 & $<0.000001$ \\
\hline
\end{tabular}

"Ratio represents the mean number of bacteria associated per PMN for the test serum divided by the mean number per PMN for its control.

$+P$ values determined by the unpaired two-tail Student's $t$-test.

Table 2. Scoring of lung lesions in mice after challenge with the FD2 or FD4 immunotypes of $P$. aeruginosa

\begin{tabular}{|c|c|c|c|c|c|c|c|c|}
\hline \multirow[t]{2}{*}{ Strain } & \multirow[t]{2}{*}{ Immunization group } & \multicolumn{5}{|c|}{$\begin{array}{l}\text { No. of mice with } \\
\text { lesions scored as": }\end{array}$} & \multirow[t]{2}{*}{$\begin{array}{l}\text { Proportion with } \\
\text { lesions } \geqslant 2+\end{array}$} & \multirow[t]{2}{*}{$P$ value } \\
\hline & & 0 & $1+$ & $2+$ & $3+$ & $4+$ & & \\
\hline \multirow[t]{4}{*}{ FD2 } & wtCPMV in alum & 3 & 0 & 2 & 9 & 13 & $24 / 27(88.9 \%)$ & - \\
\hline & CPMV-PAE5 in alum & 10 & 0 & 3 & 9 & 6 & $18 / 28(64 \cdot 3 \%)$ & $0 \cdot 03$ \\
\hline & wtCPMV in FCA/FIA & 3 & 2 & 1 & 6 & 15 & $22 / 27(81.5 \%)$ & - \\
\hline & CPMV-PAE5 in FCA/FIA & 15 & 3 & 2 & 6 & 1 & $9 / 27(33 \cdot 3 \%)$ & $0 \cdot 0004$ \\
\hline \multirow[t]{4}{*}{ FD4 } & wtCPMV in alum & 3 & 1 & 0 & 9 & 14 & $23 / 27(85 \cdot 2 \%)$ & - \\
\hline & CPMV-PAE5 in alum & 7 & 2 & 3 & 7 & 2 & $12 / 21(57 \cdot 1 \%)$ & $0 \cdot 03$ \\
\hline & wtCPMV in FCA/FIA & 3 & 0 & 1 & 11 & 6 & $18 / 21(85.7 \%)$ & - \\
\hline & CPMV-PAE5 in FCA/FIA & 21 & 5 & 3 & 14 & 4 & $21 / 47(44.7 \%)$ & $0 \cdot 0014$ \\
\hline
\end{tabular}

"Lesions were scored as follows: 0 , absence of any macroscopic lesion; $1+$, presence of one or two small lesions not exceeding $1 \mathrm{~mm}$ in diameter; $2+$, presence of three or more small lesions not exceeding $1 \mathrm{~mm}$ in diameter; $3+$, presence of a medium lesion $2-5 \mathrm{~mm}$ in diameter; $4+$, presence of a large lesion exceeding $5 \mathrm{~mm}$ in diameter.

$+P$ value was determined by Fisher's exact test.

FCA/FIA (ratio of 1.93) was used (Table 1). In parallel studies, sera from mice immunized with CPMV-PAE5 in either FCA/FIA or alum were also opsonic for FD4 (Table 1).

\section{CPMV-PAE5 immunization protects mice from $P$. aeruginosa challenge}

Mice immunized with CPMV-PAE5 in either FCA/FIA or alum were significantly protected against the development of severe $(\geqslant 2+)$ lung lesions (Table 2 ). Following challenge with FD2 in mice in which FCA/FIA was used as the adjuvant, $81.5 \%$ of those immunized with wtCPMV had severe lesions, scored as $\geqslant 2+$, whereas only $33.3 \%(P=0 \cdot 0004)$ of mice immunized with CPMV-PAE5 had severe lesions. Although protection conferred by CPMV-PAE5 was higher when FCA/FIA was used, significant protection was also seen with CPMV-PAE5 when alum was used as the adjuvant: $88.9 \%$ of mice immunized with wtCPMV had severe lesions, whereas only $64.3 \% \quad(P=0.03)$ of mice immunized with CPMV-PAE5 had severe lesions. Similar levels of protection from FD4 challenge were also seen in mice immunized with CPMV-PAE5 in FCA/FIA or alum (Table 2). A second indicator of protection afforded by the CVPs was the decrease in bacteria 
Table 3. Number of $P$. aeruginosa in lungs of mice after challenge with the FD2 or FD4 immunotypes of $P$. aeruginosa

\begin{tabular}{|c|c|c|c|c|c|}
\hline Strain & Immunization group & $\begin{array}{l}\text { No. of mice with } \\
\text { no growth/total } \\
\text { no. of mice }\end{array}$ & $P$ value & $\begin{array}{l}\text { No. of mice with } \\
<5 \times 10^{3} \text { c.f.u. in } \\
\text { lungs/total no. } \\
\text { of mice }\end{array}$ & $P$ value* \\
\hline \multirow[t]{4}{*}{ FD2 } & wtCPMV in alum & $4 / 27(14.8 \%)$ & - & $5 / 27(18.5 \%)$ & - \\
\hline & CPMV-PAES in alum & $8 / 28(28.6 \%)$ & $0 \cdot 18$ & $11 / 28(39 \cdot 3 \%)$ & 0.08 \\
\hline & wtCPMV in FCA/FIA & $7 / 26(26.9 \%)$ & - & $7 / 26(26.9 \%)$ & - \\
\hline & CPMV-PAES in FCA/FIA & $17 / 27(63 \%)$ & 0.009 & $20 / 27(74 \cdot 1 \%)$ & $0 \cdot 0007$ \\
\hline \multirow[t]{2}{*}{ FD4 } & wtCPMV in FCA/FIA & $7 / 21(33 \cdot 3 \%)$ & - & $10 / 21(47.6 \%)$ & - \\
\hline & CPMV-PAE5 in FCA/FIA & $29 / 47(61.7 \%)$ & 0.028 & $37 / 47(78.7 \%)$ & 0.012 \\
\hline
\end{tabular}

* $P$ values were determined by Fisher's exact test.

present in the lungs of immunized mice $8 \mathrm{~d}$ after challenge (Table 3). When FCA/FIA was used as the adjuvant and mice were challenged with the FD2 strain of $P$. aeruginosa, the proportion of mice whose lungs yielded no bacterial growth was increased from $26.9 \%$ in mice immunized with wtCPMV to $63 \%(P=0.009)$ in mice immunized with CPMV-PAE5. When CPMVPAE5 in alum was used, a higher (approx. double) proportion of the lungs from immunized mice also had no growth compared to mice immunized with wtCPMV in alum, but these differences were not significant (Table $3)$. A significantly $(P=0.028)$ higher number $(61.7 \%)$ of mice immunized with CPMV-PAE5 in FCA/FIA and challenged with the FD4 immunotype strain of $P$. aeruginosa (Table 3) had no bacterial growth in their lungs when compared to mice immunized with wtCPMV in FCA/FIA (33.3\%).

In mice challenged with the FD2 strain, there was a significantly $(P=0.0007)$ higher number immunized with CPMV-PAE5 in FCA/FIA $(74.1 \%)$ that had $<5 \times 10^{3}$ c.f.u. in their lungs compared to mice immunized with wtCPMV in FCA/FIA $(26.9 \%)$. When alum was used, although a higher number of CPMVPAE5-immunized mice $(39.3 \%)$ had $<5 \times 10^{3}$ c.f.u. in their lungs compared to wtCPMV-immunized mice $(18.5 \%)$, this difference was not significant $(P=0.08)$. In mice challenged with FD4 (Table 3 ) a significantly $(P=0.012)$ higher number $(78.7 \%)$ of mice immunized with CPMV-PAE5 in FCA/FIA had $<5 \times 10^{3}$ c.f.u. in their lungs compared to mice immunized with wtCPMV in FCA/FIA $(47 \cdot 6 \%)$.

The $5 \times 10^{3}$ c.f.u. cut-off has been shown previously to represent a $95 \%$ decline from the mean number of bacteria found in the lungs of challenged, control mice on day 8 after challenge in this model (Staczek et al., 1998). Since approximately $5 \times 10^{3}$ c.f.u. of $P$. aeruginosa were inoculated into the lungs at the time of challenge, this cut-off also indicates whether the inoculated bacteria were controlled or were capable of establishing an infection that resulted in a substantial increase in bacterial number within the lung.

\section{DISCUSSION}

We have demonstrated here that a chimaeric plant virus particle expressing a tandem peptide containing two linear $\mathrm{B}$ cell epitopes from $\mathrm{OM}$ protein $\mathrm{F}$ of $P$. aeruginosa (Gilleland et al., 1995) confers protection upon outbred mice when challenged with $P$. aeruginosa in a chronic model of pulmonary infection. The protection observed is mediated by the peptide 10 epitope, since immunization with CPMV-PAE5 fails to elicit antibodies to peptide 18 (Brennan et al., 1999b). The peptide 10 epitope of OM protein $\mathrm{F}$ is conserved among the various strains of $P$. aeruginosa as evidenced by antibodies directed towards peptide 10 reacting at high titre to all strains of $P$. aeruginosa tested, including strains representing each of the seven Fisher-Devlin immunotypes and over 50 clinical strains (Gilleland \& Gilleland, 1995). The four doses administered represent $\sim 5 \cdot 2 \mu \mathrm{g}$ of the tandem peptide and thus only $\sim 2.6 \mu \mathrm{g}$ of the protective peptide 10 epitope. Very low doses of OM protein $F$ peptide, when expressed on CPMV, elicited high titres of $P$. aeruginosa-specific antibody which recognized heterologous immunotypes (FD2 and FD4), opsonized the bacteria for phagocytosis by human neutrophils and conferred protection from pulmonary challenge. CVP-immunized mice had fewer severe lesions and lower numbers of bacteria in their lungs compared to mice immunized with the wild-type virus following challenge with $P$. aeruginosa. It has previously been demonstrated that KLH-conjugated protein $\mathrm{F}$ peptides (Hughes \& Gilleland, 1995) as well as killed whole $P$. aeruginosa (Cripps et al., 1994) can elicit $P$. aeruginosa-specific antibody in serum, but not in the lung, and can provide protection from $P$. aeruginosa challenge. It is most likely therefore that opsonic serum $\mathrm{IgG}$ is entering the lung and conferring protection. Indeed, clearance of $P$. aeruginosa infection from the lung was enhanced by the intravenous infusion of immune serum (Dunkley et al., 1995).

In this study, mice were immunized with CPMV-PAE5 in Freund's adjuvants since previous studies (Brennan et al., 1999b) demonstrated that immunization of mice 
with CPMV-PAE5 in Freund's adjuvants elicited the production of high titres of both peptide- and whole $P$. aeruginosa-specific $\lg G$. The more clinically applicable alum adjuvant was also tested with CPMV-PAE5 as alum is the only adjuvant currently accepted for human use (Gupka \& Siber, 1995). The titres of $P$. aeruginosaspecific IgG elicited by CPMV-PAE5, and consequently the levels of $P$. aeruginosa opsonization and the degree of protection following challenge, were substantially higher with Freund's adjuvants than with alum. This implies that more effective adjuvants must be sought for use in humans to enhance the immunogenicity of CPMV-PAE5.

The commonly used carrier molecule keyhole limpet haemocyanin (KLH) has been used previously to deliver $\mathrm{OM}$ protein $\mathrm{F}$ peptides to the immune system of both mice and rats (Gilleland \& Gilleland, 1995; Hughes \& Gilleland, 1995). Peptide 10, when coupled to KLH and given in alum, conferred protection to both rats and mice from $P$. aeruginosa infection (Gilleland \& Gilleland, 1995; Hughes \& Gilleland, 1995). In these studies, much higher levels of peptide 10 were used for immunization compared to that delivered by CPMV-PAE5 in this study. Nevertheless, it is impossible to say if CPMV is a more efficient carrier of these peptides than KLH since there were differences in the immunization regimens and adjuvants used in the different studies. A chimaeric influenza virus expressing the $\mathrm{OM}$ protein $\mathrm{F}$ peptide 10 also confers protection from $P$. aeruginosa FD4 challenge in this same mouse chronic pulmonary infection model (Staczek et al., 1998). The levels of protection achieved with the chimaeric influenza virus were lower $(55.6 \%$ of challenged mice had severe lesions) than with CPMV-PAE5 given in Freund's adjuvants $(44.7 \%$ with severe lesions, described herein). However, again it is difficult to make direct comparisons between the two viruses since the live influenza was administered using a different immunization regimen and in the absence of adjuvant. Although it is attractive to think that the use of live respiratory viruses, such as influenza virus, to deliver peptide 10 directly to the lung may serve to generate peptide-10-specific antibody in both the upper and lower respiratory tract, protection from $P$. aeruginosa infection can be achieved using vaccines that elicit protective serum $\operatorname{IgG}$ without eliciting specific antibody in the lung (Hughes \& Gilleland, 1995). There may also be safety concerns regarding the delivery of live respiratory viruses to patients with chronic lung infections, although live, attenuated influenza viruses have been shown to be safe in CF patients (Gruber et al., 1994). Such safety concerns would not be a consideration with the use of chimaeric plant virus vaccines in humans.

Although it is clear from the studies described here that for CPMV-PAE5 to function as an effective P. aeruginosa vaccine a strong adjuvant is required, the use of CPMV does have several advantages over other $P$. aeruginosa vaccine candidates. The ease of production of chimaeric plant viruses and their favourable safety profile, as well as their ability to protect against both viral (Dalsgaard et al., 1997) and bacterial (described herein) diseases, make them attractive candidates for development as vaccines against infectious disease agents.

\section{ACKNOWLEDGEMENTS}

We thank Dr Tim Jones and Fan Xu, Axis Genetics plc, for the construction of CPMV-PAE5. This work was performed under MAFF licence no. PHL 91/2275 (08/1997).

\section{REFERENCES}

Abdillahi, H. \& Poolman, J. T. (1987). Whole cell ELISA for typing Neisseria meningitidis with monoclonal antibodies. FEMS Microbiol Lett 48, 367-371.

Adams, S. E., Dawson, K. M., Gulli, K., Kingsman, S. M. \& Kingsman, A. J. (1987). The expression of hybrid HIV : ty viruslike particles in yeast. Nature 329, 68-70.

Apostolopoulos, V., Pietersz, G. A., Loveland, B. E., Sandrin, M. S. \& McKenzie, I. F. C. (1995). Oxidative/reductive conjugation of mannan to antigen selects for $\mathrm{T} 1$ or $\mathrm{T} 2$ immune responses. Proc Natl Acad Sci USA 92, 10128-10132.

Ball, J. M., Hardy, M. E., Atmar, R. L., Conner, M. E. \& Estes, M. E. (1998). Oral immunization with recombinant Norwalk virus-like particles induces a systemic and mucosal immune response in mice. J Virol 72, 1345-1353.

Brennan, F. R., Bellaby, T., Helliwell, S. M., Jones, T. D., Kamstrup, S., Dalsgaard, K., Flock, J-I. \& Hamilton, W. D. O. (1999a). Chimeric plant virus particles administered nasally or orally induce systemic and mucosal immune responses in mice. $J$ Virol 73, 930-938.

Brennan, F. R., Jones, T. D., Gilleland, L. B. \& 9 other authors (1999b). Pseudomonas aeruginosa outer membrane protein $F$ epitopes are highly immunogenic when expressed on a plant virus. Microbiology 145, 211-220.

Buratti, E., McLain, L., Tisminetzky, S., Cleveland, S. M., Dimmock, N. J. \& Baralle, E. (1998). The neutralizing antibody response against a conserved region of human immunodeficiency virus type 1 gp41 (amino acid residues 731-752) is uniquely directed against a conformational epitope. J Gen Virol 79, 2709-2716.

Buret, A. (1994). Pseudomonas aeruginosa infections in patients with cystic fibrosis, new immunomodulatory strategies. Clin Immunother 2, 261-277.

Cripps, A. W., Dunkley, M. L. \& Clancy, R. L. (1994). Mucosal and systemic immunization with killed Pseudomonas aeruginosa protect against acute respiratory infection in rats. Infect Immun $62,1427-1436$.

Dalsgaard, K., Uttenthal, A., Jones, T. D. \& 12 other authors (1997). Plant-derived vaccine protects target animals against a viral disease. Nat Biotechnol 15, 248-252.

Deprez, B., Sauzet, J.-P., Boutillon, C., Martinon, F., Tartar, A., Sergheraert, C., Guillet, J.-G., Gomard, E. \& Gras-Masse, H. (1996). Comparative efficiency of simple lipopeptide constructs for in vivo induction of virus-specific CTL. Vaccine 14, 375-382.

Dunkley, M. L., Cripps, A. W., Reinbott, R. \& Clancy, R. L. (1995). Immunity to respiratory $P$. aeruginosa infection: the role of gutderived Thelper cells and immune serum. In Advances in Mucosal Immunology, pp. 771-775. Edited by J. Mestecky, H. Tlaskalova \& J. Steriz. New York: Plenum.

Elson, C. O. \& Dertzbaugh, M. T. (1994). Mucosal adjuvants. In Handbook of Mucosal Immunology, p. 391-402. Edited by P. L. Ogra, J. Mestecky, M. E. Lamm, W. Stroher, J. R. McGhee \& J. Bienenstock. New York: Academic Press. 
Everest, P., Griffiths, P. \& Dougan, G. (1995). Live Salmonella vaccines as a route towards oral immunization. Biologicals 23, 119-124.

Gilleland, L. B. \& Gilleland, H. E., Jr (1995). Synthetic peptides representing two protective, linear B cell epitopes of outer membrane protein $\mathrm{F}$ of Pseudomonas aeruginosa elicit whole cellreactive antibodies that are functionally pseudomonad-specific. Infect Immun 63, 2347-2351.

Gilleland, H. E., Jr, Gilleland, L. B. \& Matthews-Greer, J. M. (1988). Outer membrane protein $\mathrm{F}$ preparation of Pseudomonas aeruginosa as a vaccine against chronic pulmonary infection with heterologous immunotype strains in a rat model. Infect Immun 56, $1017-1022$

Gilleland, H. E., Jr, Gilleland, L. B., Hughes, E. E. \& MatthewsGreer, J. M. (1992). Recombinant outer membrane protein $F$ of Pseudomonas aeruginosa elicits antibodies that mediate opsonophagocytic killing but not complement-mediated bacteriolysis of various strains of $P$. aeruginosa. Curr Microbiol 24, 1-7.

Gilleland, H. E., Jr, Hughes, E. E., Gilleland, L. B., MatthewsGreer, J. M. \& Staczek, J. (1995). Use of synthetic peptides to identify surface-exposed, linear B-cell epitopes within outer membrane protein F of Pseudomonas aeruginosa. Curr Microbiol 31, 279-286.

Gruber, W. C., Campbell, P. W., Thompson, J. M., Reed, G. W., Roberts, B. \& Wright, P. F. (1994). Comparison of live attenuated and inactivated influenza vaccines in cystic fibrosis patients and their families: results of a 3-year study. J Infect Dis 169, 241-247.

Gupka, R. K. \& Siber, G. R. (1995). Adjuvants for human vaccines - current status, problems and future prospects. Vaccine $13,1263-1276$.

Hughes, E. E. \& Gilleland, H. E., Jr (1995). Ability of synthetic peptides representing epitopes of outer membrane protein $F$ of Pseudomonas aeruginosa to afford protection against $P$. aeruginosa in a murine acute pneumonia model. Vaccine 13, 1750-1753.

Hughes, E. E., Gilleland, L. B. \& Gilleland, H. E., Jr (1992). Synthetic peptides representing epitopes of outer membrane protein $F$ of Pseudomonas aeruginosa that elicit antibodies reactive with whole cells of heterologous immunotype strains of $P$. aeruginosa. Infect Immun 60, 3497-3503.

Jahn-Schmid, B., Siemann, U., Zenker, A. \& 7 other authors (1997). Bet $v 1$, the major birch pollen allergen, conjugated to crystalline bacterial cell surface proteins, expands allergen-specific $T$ cells of the Th1/Th0 phenotype in vitro by induction of IL-12. Int Immunol 9, 1867-1875.

Klinman, D. M., Takeno, M., Ichino, M., Gu, M., Yamshchikov, G., Mor, G. \& Conover, J. (1997). DNA vaccines: safety and efficacy issues. Springer Semin Immunopathol 19, 245-256.

Lovgren, K., Kaberg, H. \& Morein, B. (1990). An experimental influenza subunit vaccine (ISCOM): induction of protective immunity to challenge infection in mice after intranasal or subcutaneous administration. Clin Exp Immunol 82, 435-440.

McLain, L., Porta, C., Lomonossoff, G. P., Durrani, Z. \& Dimmock,
N. J. (1995). Human immunodeficiency virus type 1 neutralizing antibodies raised to a gp41 peptide expressed on the surface of a plant virus. AIDS Res Hum Retroviruses 11, 327-334.

McLain, L., Durrani, Z., Wisniewski, L. A., Porta, C., Lomonossoff, G. P. \& Dimmock, N. J. (1996). Stimulation of neutralizing antibodies to human immunodeficiency virus type 1 in three strains of mice immunized with a 22 amino acid peptide of gp41 expressed on the surface of a plant virus. Vaccine 14, 799-810.

Montgomery, D. L., Donnelly, J. J., Shiver, J. W., Liu, M. A. \& Ulmer, J. B. (1994). Protein expression in vivo by injection of polynucleotides. Curr Opin Biotechnol 5, 505-510.

Porta, C., Spall, V. E., Loveland, J. E., Johnson, J. E., Barker, P. J. \& Lomonossoff, G. P. (1994). Development of cowpea mosaic virus as a high-yielding system for the presentation of foreign peptides. Virology 202, 949-955.

Reed R. C., Louis-Wileman, V., Cosmai, E. V., Fang, S., Jue, D. L., Wohlhueter, R. M., Hunter, R. L. \& Lal, A. A. (1997). Multiple antigen constructs (MACs): induction of sterile immunity against sporozoite stage of rodent malaria parasites, Plasmodium berghei and Plasmodium yoelii. Vaccine 15, 482-488.

Resnick, D. A., Smith, A. D., Geisler, S. C., Zhang, A., Arnold, A. \& Arnold, G. F. (1995). Chimeras from a human rhinovirus 14human immunodeficiency virus type 1 (HIV1) V3 loop seroprevalence library induce neutralizing responses against HIV-1. J Virol 69, 2406-2411.

Rose, C. S. P. \& Evans, D. J. (1991). Poliovirus antigen chimeras. Trends Biotechnol 9, 415-421.

Staczek, J., Gilleland, H. E., Jr, Gilleland, L. B., Harty, R. N., GarciaSastre, A., Engelhardt, O. G. \& Palese, P. (1998). A chimeric influenza virus expressing an epitope of outer membrane protein F of Pseudomomas aeruginosa affords protection against challenge with $P$. aeruginosa in a murine model of chronic pulmonary infection. Infect Immun 66, 3990-3994.

Sutter, G. \& Moss, B. (1992). Non-replicating vaccinia vector efficiently expresses recombinant genes. Proc Natl Acad Sci USA 89, 10847-10851.

Usha, R., Rohll, J., Spall, V. E., Shanks, M., Maulie, A. J., Johnson, J. E. \& Lomonossoff, G. P. (1993). Expression of an animal virus antigenic site on the surface of a plant virus particle. Virology 197, 366-374.

Vlacha, V., Forman, E. N., Mirond, D. \& Peter, G. (1996). Recurrent thrombocytopenic purpura after repeated measles/mumps/ rubella vaccination. Pediatrics $\mathbf{9 7}, \mathbf{7 3 8}-\mathbf{7 3 9}$.

WHO (1990). Potential use of live viral and bacterial vectors for vaccines. WHO meeting, Geneva, 19-22 June, 1989. Vaccine 8, $425-437$.

Zhang, S., Graebaer, L. A., Helling, F., Ragupathi, G., Adluri, S., Lloyd, K. O. \& Livingston, P. O. (1996). Augmenting the imunogenicity of synthetic MUC1 peptide vaccines in mice. Cancer Res 56, 3315-3319.

Received 18 January 1999; revised 3 April 1999; accepted 15 April 1999. 